\title{
The Global Interaction of Comets with the Solar Wind
}

\author{
K. R. Flammer \\ Department of Electrical and Computer Engineering \\ University of California, San Diego \\ La Jolla, CA 92093, USA
}

\begin{abstract}
The global interaction of the solar wind with a comet as it orbits the Sun is reviewed. After a brief survey of the flow transition regions observed at comet Halley is presented, theoretical models are given for the cometocentric distance of the bow shock, the cometopause, and the ionopause. In addition, predictions are made as to what heliocentric distance these boundaries should form at. The results of these models are compared with the in situ observations at comet Halley.
\end{abstract}

\section{Introduction}

Unlike the atmospheres of planets and asteroids, the atmosphere of a comet varies dramatically in the comet's elliptical orbit around the Sun, and thus the nature of the atmosphere's interaction with the solar wind changes considerably with heliocentric distance. The crucial parameters that determine the nature and size of the comet-solar wind interaction region are the cometary neutral production rate and the resulting cometary ion density. In the case of the solar wind interaction with a well-developed cometary atmosphere, (i.e., $d \leq 2 \mathrm{AU}$ for a Halley-type comet), an outer bow shock diverts the supersonic solar wind around the cometary atmosphere, and the shocked, mass-loaded plasma is separated from the comet nucleus and surrounding comet plasma by a tangential discontinuity interface. At larger heliocentric distances, $d \approx 4 \mathrm{AU}$, the characteristic flow boundaries have not yet formed. In this case the unshocked solar wind flow continues to pick up cometary ions and become increasingly mass-loaded towards the comet nucleus, while the interplanetary magnetic field is slightly enhanced. Beyond $\approx 5 \mathrm{AU}$, an $\mathrm{H}_{2} \mathrm{O}$-dominated comet has no significant atmosphere, so the solar wind directly impacts the inactive comet nucleus.

In this review, we will describe the global interaction of the solar wind with a comet over a range of heliocentric distances, focusing on the macroscopic flow patterns and characteristic boundaries that form in the solar wind flow due to the presence of the outgassing comet. Quantitative models will be given for the location of the flow transition regions relative to the comet nucleus and the heliocentric distance where the various boundaries should form. The in situ observations at comets Giacobini-Zinner and Halley confirmed the large-scale picture of the solar wind interaction with comets at close heliocentric distances. The dominant processes leading to the formation of the two main flow boundaries-the bow shock and the tangential discontinuity (referred to as the ionopause) are fairly well understood, and their positions relative to the comet nucleus can be predicted from the global models. The boundary or transition region that lies between the bow shock and the ionopause, the "cometopause" or "collisionopause," is less understood at present. What was also revealed by the spacecraft data is the importance and complexity of the collective plasma processes in the comet-solar wind interaction; namely, the hydromagnetic turbulence and the wave-particle interactions. We will only briefly mention the kinetic aspect 
of the solar wind interaction with comets and refer the reader to relevant studies for more detail. The primary goal of the global models presented here is to predict the heliocentric and cometocentric variation of the various flow transition regions.

\section{Global Interaction Close to the Sun}

Ever since the pioneering work of Biermann et al. (1967), almost all of the studies of the comet-solar wind interaction have dealt with the case of a well-developed comet close to the Sun (d $\leq 1$ AU) (e.g., Schmidt and Wegmann, 1976, 1982; Wallis, 1973; Wallis and Dryer, 1976; Houpis and Mendis, 1980; Galeev et al., 1984; for an extensive review, see Mendis et al., 1985). The overall morphology of this interaction is shown schematically in Figure 1. Due to the negligible gravity of the small cometary nucleus, the sublimating

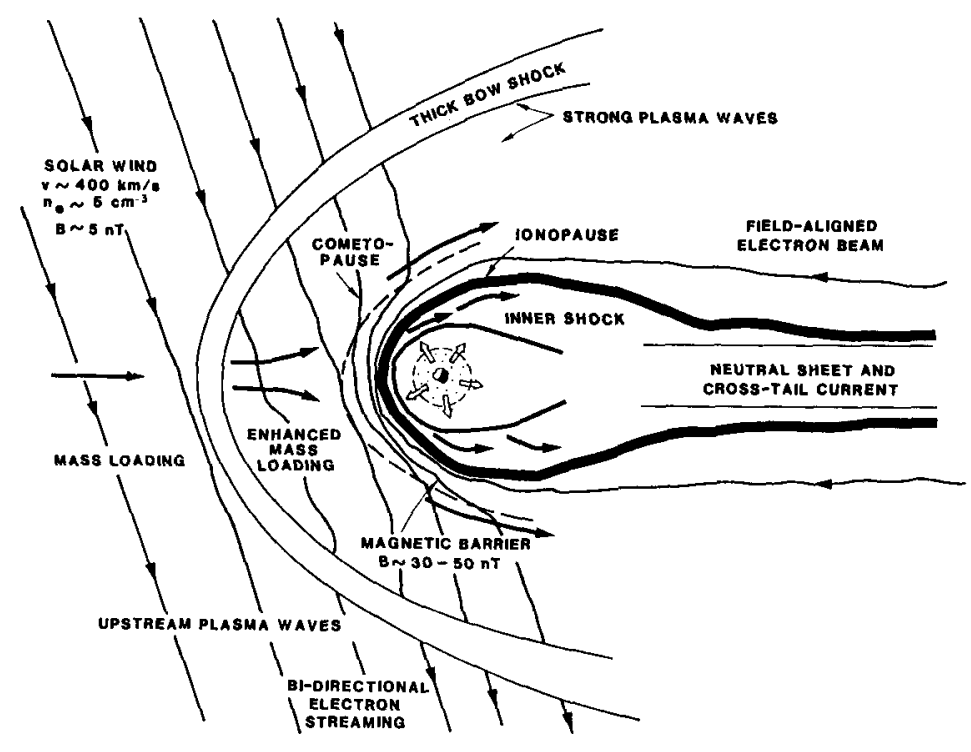

Figure 1. Schematic representation of the global morphology of the solar wind interaction with the cometary atmosphere, showing the various discontinuities in the flow pattern.

gases expand supersonically outward from the nucleus and interact with the solar wind over a scale that is 5 to 6 orders of magnitude greater than the size of the nucleus $(\approx 1$ to $20 \mathrm{~km}$ in diameter). Heavy cometary neutral species $\left(\mathrm{H}_{2} \mathrm{O}, \mathrm{OH}, \mathrm{O}\right)$ are ionized by solar ultraviolet radiation or charge exchange with the solar wind ions and are assimilated into the magnetized solar wind. The continuous mass loading of the inflowing solar wind by the newly created ions causes the solar wind to decelerate and heat up. Continuous deceleration of the solar wind flow by mass-loading is possible only as long as the mean molecular weight of the plasma remains at less than a critical value. Before this critical value is reached, a weak shock forms ahead of the comet. Downstream from the shock, the mass-loaded 


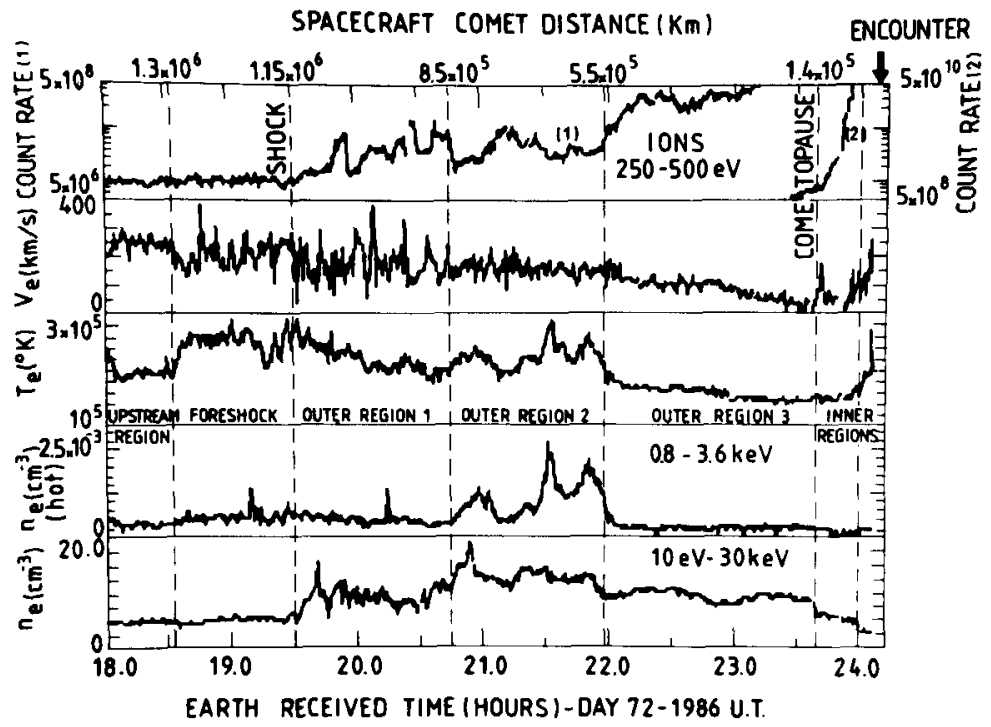

Figure 2. Electron parameters measured by Giotto on its inbound trajectory at comet Halley. From top to bottom: total electron count rate, electron velocity, electron temperature, density of electrons in energy range $0.8-3.6 \mathrm{keV}$, density of electrons in range $10 \mathrm{eV}$ to 30 $\mathrm{keV}$. (from Reme, 1990)

subsonic solar wind continues to interact with the comet atmosphere, penetrating into a region of ever increasing neutral density. The outflowing cometary neutrals play a crucial role in collisionally slowing down the inflowing solar wind. Strong deceleration occurs at a boundary we term the "collisionopause" or "cometopause," where significant momentum is transferred from the outflowing cometary neutrals to the solar wind ions by collisions. Inside this transition region, collisions dominate and the solar wind decelerates rapidly and cools due to charge exchange processes with the less energetic cometary neutrals, while the magnetic field compresses to form a magnetic barrier region. A tangential discontinuity interface forms at the inner edge of the magnetic barrier region and separates the two plasmas: the purely cometary plasma and the mass-loaded solar wind plasma. An inner shock which decelerates the supersonically outward flowing cometary ions and diverts them into the tail has been suggested and discussed theoretically, (Wallis and Dryer, 1976; Houpis and Mendis, 1980; Korosmezey et al., 1986; Omidi et al., 1989), although such a structure has not yet been observed. Alternatively, a recombination layer has been suggested by Cravens (1989) to accomplish the same purpose.

This depiction of the global interaction was largely confirmed by the comet Halley missions, although in addition to the predicted boundary regions which will be discussed in this chapter: the bow shock, the collisionopause/cometopause and the ionopause, the ob- 


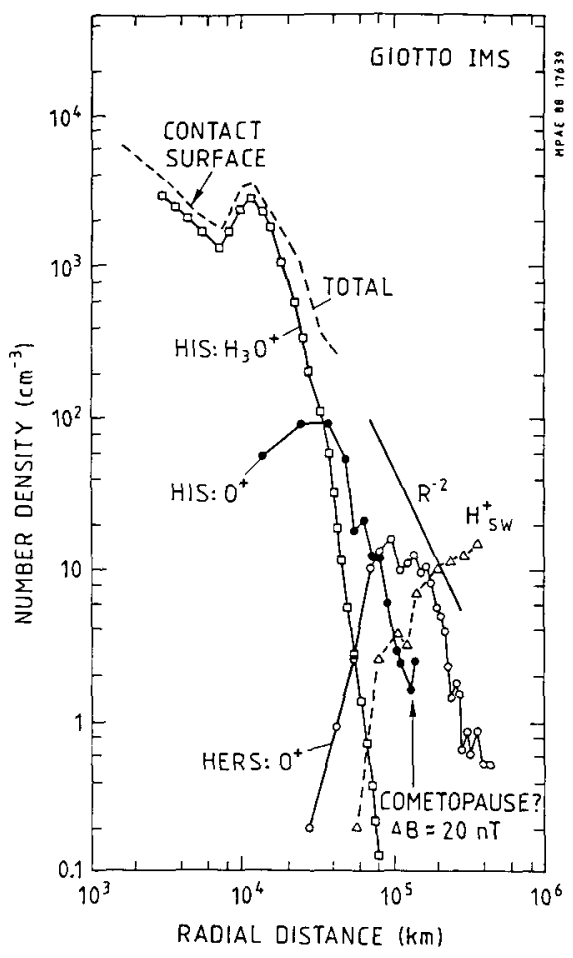

Figure 3. Number density profiles of water group ions and solar wind protons measured by the Giotto ion mass spectrometer. (from Ip, 1989)

servations indicate that there may be additional features of this interaction which are not yet understood theoretically. The ESA Giotto spacecraft flew in front of comet Halley on the sunward side and crossed the bow shock, the collisionopause/cometopause and the cometary ionopause. A weak shock was observed by the Giotto electron electrostatic analyser (EESA) at a radial distance of $\approx 1.15 \times 10^{6} \mathrm{~km}(1922 \mathrm{UT})$, (see Figure 2), where the flow speed decreased and the electron density increased in magnitude and in the degree of its fluctuations. Inside the bow shock, in the cometosheath region, the flow continues to decelerate as the ion pickup increases and the plasma becomes more dominated by cometary ions. An interesting but poorly understood region was observed between $\approx 8.5 \times 10^{5} \mathrm{~km}$ and $5.5 \times 10^{5} \mathrm{~km}$ labelled "outer region 2" in Figure 2, and also rather appropriately called the "mystery region". In the mystery region itself, there is a high flux of energetic electrons which disappear abruptly as the region is exited. At this point also, both the total ion density and velocity decrease, while the ion temperature increases (Reme, 1990). Because similar features were observed in the outbound Giotto observations as well as the inbound Vega 2 observations, the mystery region might be a consequence of the comet-solar wind 
interaction rather than a transient phenomenon.

Closer in towards the nucleus, at a cometocentric distance of $\approx 1.39 \times 10^{5} \mathrm{~km}$, there was a sudden decrease in the density of electrons with energies greater than $10 \mathrm{eV}$ (see bottom panel of Figure 2), as well as an increase in the cold ion density (Balsiger et al., 1987), and an increase in the magnetic field strength from $\approx 6$ to $26 \mathrm{nT}$ (Neubauer et al., 1986;1987). This transition region has been indentified with the cometopause or collisionopause. Another interesting feature, a density peak, was observed in the Giotto ion data at $\approx 10,000 \mathrm{~km}$ from the nucleus, as shown in Figure 3. This density enhancement was also seen in the Vega 1 data, both inbound and outbound (Vaisberg et al., 1988). Although this density peak was first called a "pile-up" region, (Balsiger et al., 1986), it is now believed that the discontinuity in the density variation could be due to a change in the electron temperature which modifies the electron/ion recombination rate (Ip et al., 1987). Inside $10,000 \mathrm{~km}$, the concentration of the dominant cometary ions, $\mathrm{H}_{2} \mathrm{O}^{+}$and $\mathrm{H}_{3} \mathrm{O}^{+}$, continuously increases towards the nucleus, however at a distance of about $4700 \mathrm{~km}$, the ion temperature dropped from $\approx 2600^{\circ} K$ to $\approx 450^{\circ} K$, (Balsiger et al., 1987 ), and a sudden onset of a $1 \mathrm{~km} / \mathrm{sec}$ flow velocity outwards from the nucleus was observed as shown in Figure 4. These results

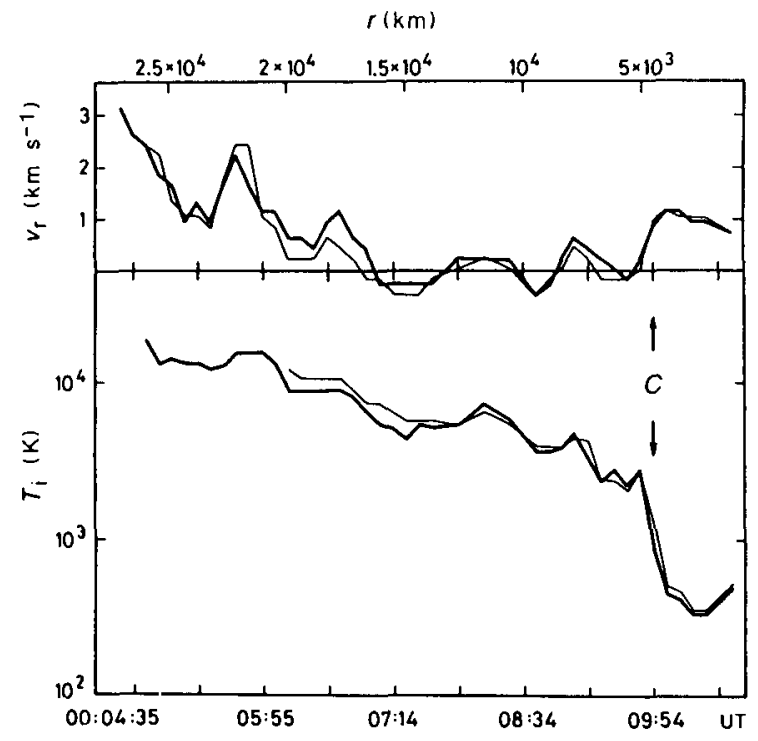

Figure 4. Radial velocity and temperature of ions with mass/charge ratio of 18 (heavy line) and 19 (lighter line) amu/charge measured inbound by Giotto (from Balsiger et al., 1986).

indeed confirmed the existence of a tangential discontinuity interface (TD), also referred to as the cometary ionopause, which separates the inflowing, contaminated solar wind and the outflowing purely cometary plasma. Even more remarkable were the Giotto magnetometer results shown in Figure 5 (Neubauer et al., 1986), which measured a dramatic drop in the 


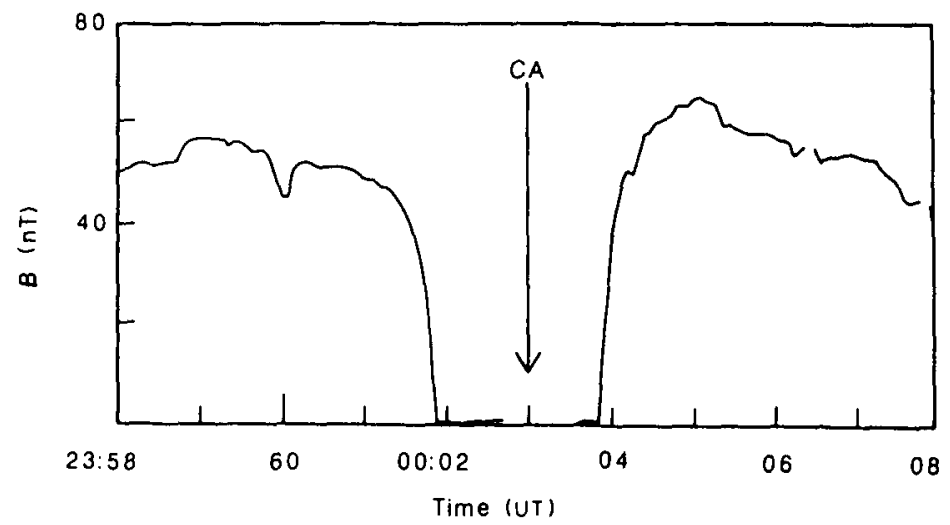

Figure 5. Magnetic field magnitude versus time measured by the magnetometer onboard Giotto (from Neubauer et al., 1986).

magnetic field to zero at a cometocentric distance of about $4600 \mathrm{~km}$ inbound, and 3900 $\mathrm{km}$, outbound. In the following sections, we present simple models for the cometocentric distance of the bow shock, the collisionopause and the ionopause, and make predictions concerning at what heliocentric distance these boundaries will form.

\subsection{NEUTRAL PRODUCTION RATE}

Since all of the boundaries that form in the interaction of the comet plasma with the solar wind plasma depend explicitly on the comet neutral production rate, $Q_{n}$, we require an expression relating the production rate and the heliocentric distance, $d$. Assuming a fast spinning, isothermal, spherical nucleus, neglecting heat conduction into the nucleus interior, and ignoring attenuation or scattering due to the entrained dust, the energy balance equation at the comet surface is:

$$
\frac{J\left(1-A_{B}\right)}{4 d^{2}}=\epsilon_{s} \sigma T_{s}^{4}+\frac{L_{M} Z f}{N_{A}},
$$

where $J$ is the solar constant, $A_{B}$ is the bolometric albedo, $d$ is the heliocentric distance in $\mathrm{AU}, \epsilon_{s}$ is the emissivity, $\sigma$ is the Stefan-Boltzmann constant, $L_{m}$ is the latent heat of sublimation per mole of surface ice, $Z$ is the flux of sublimating molecules, $N_{A}$ is Avogadro's number, and $f$ is the fraction of surface area covered with ice. Assuming that the sublimating molecules behave as a perfect gas, the Clausius-Clapeyron equation determines the 
steady state surface number density $n_{s}$ and is given by

$$
n_{s}=n_{o} \frac{T_{o}}{T_{s}} \exp \left[\frac{-L_{M}}{k N_{A}}\left(\frac{1}{T_{s}}-\frac{1}{T_{o}}\right)\right],
$$

where $n_{o}$ and $T_{o}$ are reference quantities for the ice in question (for $\mathrm{H}_{2} \mathrm{O}$ ice, $n_{o}=1.94 \times$ $10^{19} \mathrm{~cm}^{-3}, T_{o}=373^{\circ} \mathrm{K}, L_{M}=5.0 \times 10^{11} \mathrm{erg} / \mathrm{mole}$ ) and $k$ is Boltzmann's constant. Finally, we note that the gas production rate is given by

$$
Q_{n}=4 \pi R_{n}^{2} Z=4 \pi R_{n}^{2} V_{s} n_{s} f
$$

where $R_{n}$ is the nucleus radius and $V_{s}$ is the expansion speed from the surface, which we may assume to be equal to the local sonic speed:

$$
V_{s}=\sqrt{\gamma_{n} k T_{s} / m_{n}}
$$

where $\gamma_{n}$ is the ratio of specific heats for the sublimating neutrals and $m_{n}$ is the neutral mass. Solving Equations (1) and (2) together with (3) and (4) numerically gives the production rate $Q_{n}$, the surface temperature $T_{s}$ and the surface number density $n_{s}$, as a function of the heliocentric distance, $d$. The heliocentric variation of $Q_{n}$ is shown in Figure 6 using comet parameters that give a production rate that agrees with the measured value of comet Halley at $0.89 \mathrm{AU}$ of $Q_{n} \approx 6.9 \times 10^{29} \mathrm{~mol} / \mathrm{sec}$.

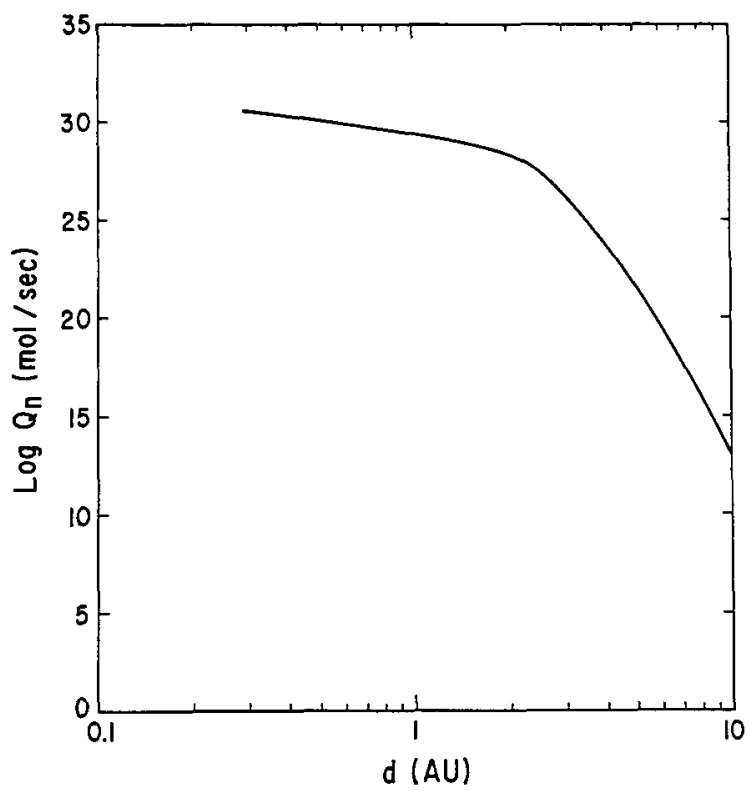

Figure 6. Heliocentric variation of $Q_{n}$ for an $\mathrm{H}_{2} \mathrm{O}$ dominated comet. 


\subsection{BOW SHOCK}

The mechanism for the formation of bow shocks at comets is quite unique among solar system objects. Planets with strong magnetic fields (e.g., Earth and Jupiter), and unmagnetized planets with dense atmospheres (e.g., Venus) form impenetrable boundaries that require an outer bow shock to divert the supersonic solar wind flow around the obstacle. The cometary bow shock, on the other hand, forms purely as a result of the mass-loading process. As first shown by Biermann et al. (1967), steady-state mass-loading of the solar wind by the cometary ions proceeds only as long as the normalized mass flux, $\hat{x}=\left(\rho u / \rho_{\infty} u_{\infty}\right)$ (where the subscript $\infty$ refers to quantities far from the comet in the undisturbed solar wind), remains at less than a critical value. Before this value is attained, a shock wave moves upstream from the comet to divert the flow around the cometary atmosphere.

The steady-state solar wind flow with mass-loading due to the cometary ions can be described by the following magnetohydrodynamic conservation equations valid along the Sun-comet axis (Biermann et al., 1967):

$$
\begin{gathered}
\frac{d}{d r}(\rho u)=\frac{m_{i} n_{n}}{\tau_{i}} \\
\frac{d}{d r}\left(\rho u+P+\frac{B^{2}}{8 \pi}\right)=0 \\
\frac{d}{d r}\left(u\left(\frac{\rho u^{2}}{2}+\frac{P \gamma}{\gamma-1}+\frac{B^{2}}{4 \pi}\right)\right)=0
\end{gathered}
$$

where $\rho, u, P, B$, and $\gamma$ are, respectively, the mass density, hydrodynamical velocity, thermal pressure, magnetic field strength (which is assumed to be perpendicular to the flow), and ratio of specific heats of the inflowing solar wind plasma, $r$ is the distance from the comet nucleus, and $n_{n}, m_{i}$, and $\tau_{i}$ are the number density, average ion mass, and ionization time scale of the cometary neutrals. The cometary neutral density is found from solving the continuity equation, assuming a spherically symmetric outflow at a constant neutral expansion speed $V_{n}$ and allowing for the loss of neutrals due to photoionization with a characteristic time scale of $\tau_{i}$, giving:

$$
n_{n}=\frac{Q_{n}}{4 \pi r^{2} V_{n}} \exp \left(-r / V_{n} \tau_{i}\right)
$$

Integrating Equation (5) using Equation (8) gives an expression for $\hat{x}$ as a function of the cometocentric distance $r$ (e.g., Galeev et al., 1985),

$$
\hat{x}=1+\frac{Q_{n} m_{i}}{4 \pi V_{n}^{2} \tau_{i}^{2} \rho_{\infty} u_{\infty}} \Psi\left(\frac{r}{V_{n} \tau_{i}}\right)
$$

where

$$
\Psi(\xi)=\xi^{-1} \exp (-\xi)+E_{1}(-\xi)
$$

and

$$
E_{1}(-\xi)=\int_{-\infty}^{-\xi} e^{z} z^{-1} d z
$$


Integrating the momentum and energy equations (6) and (7), assuming that the solar wind flow remains supersonic and super-Alfvénic so that the magnetic terms may be neglected, we find that

$$
\hat{u}=\frac{u}{u_{\infty}}=\frac{1}{\hat{x}} \frac{\gamma}{\gamma+1}\left[1 \pm \sqrt{1-\frac{\gamma^{2}-1}{\gamma^{2}} \hat{x}}\right] .
$$

Thus, in this treatment, steady-state mass-loading is possible only as long as

$$
\hat{x}=\frac{\rho u}{\rho_{\infty} u_{\infty}} \leq \frac{\gamma^{2}}{\gamma^{2}-1} .
$$

The singularity in the solution to equation (10) implies that a shock wave will form ahead of the comet to insure that the critical value for the normalized mass flux is not exceeded.

Originally, the cometary bow shock was believed to be strong, with a Mach number $(M)$ of about 10, analogous to the case of the Earth's bow shock (Biermann et al., 1967). Wallis (1973), however, suggested that because the upstream solar wind plasma is heated and decelerated due to mass-loading, the cometary bow shock should be weak, with $M=2$ or even nonexistent due to charge-exchange cooling (Wallis and Dryer, 1985). Subsequent numerical simulations (Schmidt and Wegmann, 1982; Baranov et al., 1986, Ogino et al., 1988) for a Halley-type comet at $1 \mathrm{AU}$ have shown that a weak collisionless shock forms when the contaminated solar wind Mach number decreases to $\approx 2$. To determine the value of the normalized mass flux at an $M=2$ shock, we express $\hat{x}$ as a function of the Mach number $M=u / c_{s}=u / \sqrt{\gamma P / \rho}$

$$
\hat{x}=\frac{\gamma^{2}}{\gamma^{2}-1} \frac{M^{2}\left[M^{2}(\gamma-1)+2\right]}{\left(M^{2} \gamma+1\right)^{2}}
$$

where we have assumed that $M^{2} \ll M_{\infty}^{2}$.

The "pick-up" of cometary ions by the solar wind and resulting mass-loading of the solar wind is the fundamental process governing the shock formation. The nature of the pick-up process depends upon the orientation of the solar wind flow velocity to the interplanetary magnetic field (IMF). When the IMF is orthogonal to $u$, the pick-up occurs through the macroscopic fields: the magnetic field and motional electric field $(=u \times B / c)$ (Omidi and Winske, 1987; Galeev, 1986). The newly created ions gyrate around the local magnetic field with a gyro-velocity $V_{s w}$ relative to the convected magnetic field, retaining their magnetic moment at the point of origin. In this case, the adiabatic approximation is used to describe the picked-up cometary ions and $\gamma$ may be taken to be 2 in Equation (12), so that an $M=2$ shock occurs when

$$
\hat{x}_{M=2}=\frac{32}{27} \approx 1.185 \text {. }
$$

When the solar wind flows obliquely to the magnetic field, the coupling between the solar wind and the cometary ions is dominated by the microscopic fields generated by plasma instabilities. When the magnetic field is inclined at an angle $\theta$ to the solar wind velocity, the cometary ions drift along $B$ with a velocity $V_{\|}=V_{s w} \cos \theta$ relative to the solar wind and gyrate around the field line with $V_{\perp}=V_{s w} \sin \theta$. The beaming of this gyrotropic ring distribution is subject to various plasma instabilities (Galeev 1986; 1987, Winske et al., 1985, Sagdeev et al., 1986, Wu and Davidson, 1972), which pitch-angle scatter these ions 
into a shell distribution. In the limit of strong pitch-angle scattering and rapid isotropization of the velocity distribution, $\gamma$ may be taken as $5 / 3$, and in this case we find from Equation (12) that

$$
\hat{x}_{M=2}=1.323 .
$$

Comparing Equations (13) and (14), we see that a $M=2$ shock will form closer to the cometary nucleus when the solar wind flow is inclined to the interplanetary magnetic field and strong pitch-angle scattering results than when the flow is normal to the magnetic field and the adiabatic condition is a reasonable assumption.

When the comet is sufficiently far from the Sun, the cometary atmosphere will be so tenous that the solar wind will penetrate all the way up to the nucleus without accreting enough heavy cometary ions to invalidate the inequality (Equation (11)) corresponding to critical mass-loading. Equation (9) can be solved for the distance to the bow shock along the Sun-comet axis, $R_{s}$, for a specified value of the normalized mass flux at the shock $\hat{x}_{s}$. Assuming the $R_{s} \ll V_{n} \tau_{i}$, a simple expression for $R_{s}$ results:

$$
R_{s}=\frac{Q_{n} m_{i}}{4 \pi V_{n} \tau_{i} \rho_{\infty} u_{\infty}\left[\left(\hat{x}_{s}\right)-1\right]}
$$

To compare the predicted bow shock standoff distance given by Equation (15) with the Giotto observations at comet Halley, we use the following measured parameters- $n_{\infty}=8.6$ $\mathrm{cm}^{-3}, V_{s w}=350 \mathrm{~km} / \mathrm{sec}, Q_{n}=6.9 \times 10^{29} \mathrm{~mol} / \mathrm{sec}, V_{n} \tau_{i}=2 \times 10^{6} \mathrm{~km}$ - to determine $R_{s 1}\left(\hat{x}_{s}=1.323\right)$ for the case of strong pitch-angle scattering, and $R_{s 2}\left(\hat{x}_{s}=1.185\right)$ for the adiabatic case. Assuming a parabola-shaped shock with a flaring ratio $2.15 R_{s}$ (Galeev, 1986; Mendis et al., 1986), Figure 7 shows the Giotto trajectory, which was at an angle of $107^{\circ}$ to the Sun-comet axis, and the calculated position of the two paraboloidal shocks. The Giotto inbound bow shock crossing lies in between the shock locations predicted by the two models.

Figure 8 shows the heliocentric variation of the subsolar bow shock radius given by Equation (15), where $R_{s 1}$ is based on the isotropization approximation (using $\gamma=5 / 3$ in Equation (12)), and $R_{s 2}$ is based on the adiabatic assumption (using $\gamma=2$ in Equation(12)), and considering a $M=2$ shock in both cases. (For these calculations and those that follow, a comet with a production rate similar to comet Halley is assumed). In determining at what heliocentric distance the bow shock is expected to form, the shock thickness must be taken in account. Galeev et al. (1984), using a particle in cell code to simulate the introduction of a cometary ion source into the magnetized solar wind plasma, have shown that the shock front thickness, for quasi-perpendicular shocks, has a scale on the order of the Larmor radius of a cometary ion (approximately $10^{4} \mathrm{~km}$ ), in comparison to the Earth's bow shock, which has a thickness on the order of a proton gyro-radius $(\approx 100 \mathrm{~km})$. The shock structure has further been discussed by Galeev et al. (1985) as consisting of a viscous subshock with a characteristic thickness on the order of the solar wind proton gyro-radius, $L_{p}$, where the solar wind protons are decelerated, followed by a much broader isotropization region with a scale length of the order on the cometary ion gyro-radius, $L_{i s}$. In a detailed numerical study using a hybrid particle code, Omidi and Winske (1986) also conclude that the macroscopic structure of a quasi-perpendicular shock at comet Halley is determined by the gyro-radius of the dominant $O^{+}$ions at that distance from the nucleus. If the cometary bow shock is quasi-parallel, it will be more similar to cosmic ray diffusive shocks (Sagdeev 


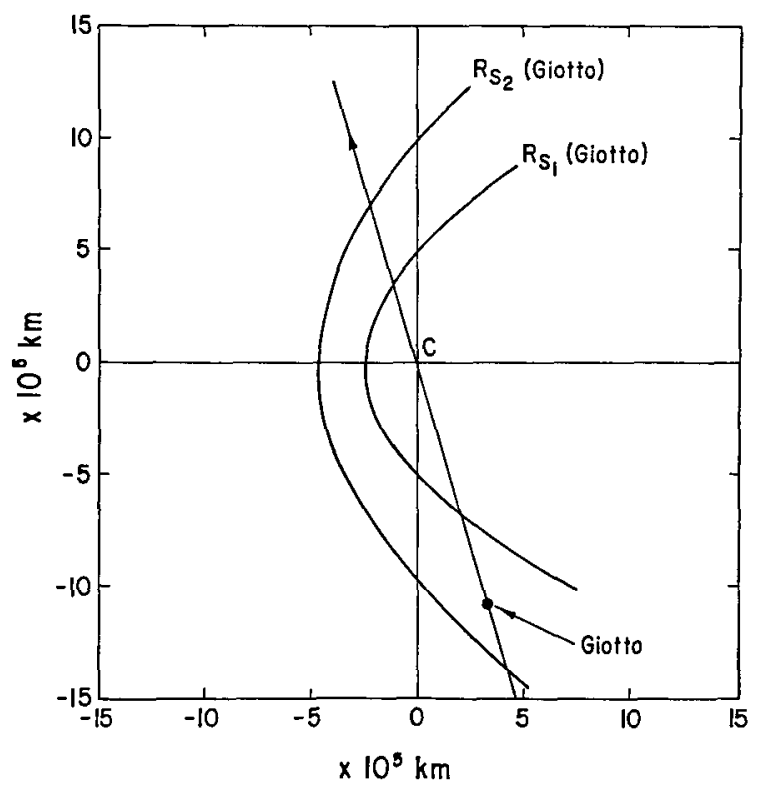

Figure 7. Giotto spacecraft trajectory and measured bow shock crossing point shown with the calculated paraboloidal shocks predicted from both the adiabatic and strong pitch-angle scattering models.

et al., 1986) and have a thickness on the order of the diffusion length, or $\approx L_{i s}$. Both quasiperpendicular and quasi-parallel bow shocks were observed at comet Halley (see Galeev, 1986).

In order to calculate the cometary ion and solar wind proton gyro-radii at the shock, we use the average solar wind magnetic field strength as predicted by the Parker spiral model:

$$
B_{\infty}=6\left(\frac{1}{2}\left(\frac{1}{d^{2}}+\frac{1}{d^{4}}\right)\right)^{\frac{1}{2}} n T
$$

and the following empirical expression for the variation of the solar wind proton temperature with heliocentric distance from 1 to $10 \mathrm{AU}$ :

$$
T_{p \infty}=8.0 \times 10^{4} d^{-0.7} K
$$

given by Gazis and Lazarus (1982). The variation of the proton temperature upstream from the shock is

$$
T_{p 1}=T_{p \infty} \hat{P} / \hat{n}
$$

where $\hat{P}$ and $\hat{n}$ are the normalized pressure and density in the mass-loaded supersonic solar wind flow. Assuming that the solar wind flow velocity remains perpendicular to the magnetic field upstream from the shock and the frozen-in condition for the magnetic field 


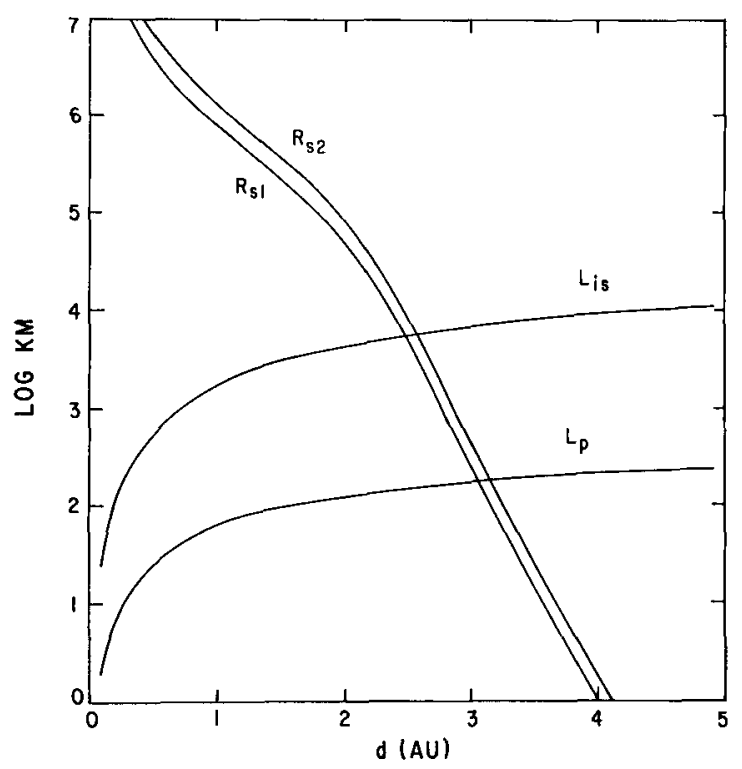

Figure 8. Heliocentric variation of the bow shock distances $R_{s 1}$ and $R_{s 2}$ for the pitch-angle scattering and adiabatic models, respectively, and the cometary ion and proton Larmor radii.

holds, (i.e., $u B=$ constant), we obtain the following expression for the proton and ion Larmor radii, respectively, at the shock front:

$$
L_{p}=\frac{c \sqrt{m_{p} k T_{p 1}}}{e B_{1}}=\frac{c \hat{u}_{1}}{e B_{\infty}} \sqrt{m_{p} k T_{p 1}}
$$

and

$$
L_{i s}=\frac{m_{i} u_{2} c}{e B_{2}}=\frac{m_{i} u_{\infty} \hat{u}_{2}^{2} c}{e B_{\infty}},
$$

where subscripts 1 and 2 refer to quantities upstream and downstream of the shock.

The condition for the formation of a fully developed quasi-perpendicular shock is

$$
R_{s} \geq L_{i s}
$$

whereas the condition for the formation of a viscous subshock is

$$
R_{s} \geq L_{p}
$$

The heliocentric variation of $L_{i s}$ and $L_{p}$ is shown in Figure 8. Also shown is the variation of the $M=2$ subsolar shock distances $R_{s 1}$ and $R_{s 2}$, where the subscript 1 corresponds to the pitch-angle scattering model and subscript 2 corresponds to the adiabatic model. From these, we find that a subshock satisfying Equation (22) can be expected for a Halley-type 
comet for $d \leq 3.06$ to $3.2 \mathrm{AU}$, and a fully developed shock satisfying Equation (21) for $d \leq$ 2.48 to $2.6 \mathrm{AU}$ (with the smaller distance corresponding to the pitch-angle scattering model and the larger' distance to the adiabatic model).

\subsection{COLLISIONOPAUSE/COMETOPAUSE}

Inside the bow shock, a transition occurs between the collisionless, mass-loaded solar wind plasma flow and a flow dominated by collisions with the expanding cometary neutral molecules. This boundary region has been termed the "collisionopause" by some authors (e.g., Neugebauer et al., 1985; Mendis and Flammer, 1984; Mendis et al., 1989), the "cometopause" by others (e.g., Gringauz et al., 1986a, b). The physical mechanisms responsible for this flow transition region are poorly understood at present and it is likely that many competing processes are involved (e.g., see Ip, 1989). Most authors agree that this intermediate boundary separates the cometary sheath region, dominated by fast-moving solar wind protons, from the region dominated by compressed interplanetary magnetic fields and slow cometary ions. The VEGA instruments at comet Halley observed a rather sudden drop in the solar wind proton density, while the cometary ion density increased rapidly near $10^{5} \mathrm{~km}$ from the nucleus over a fairly short distance $\approx 10^{4} \mathrm{~km}$ (Gringauz et al., 1986a). The boundary separating the fast solar wind plasma from the slower cometary plasma was found to be much more diffuse during the Giotto encounter (Balsiger et al., 1986; Amata et al., 1987). Also near this location, the Giotto magnetometer observed a sudden jump in the magnetic field of $\approx 20 \mathrm{nT}$ (Neubauer, 1986, 1987), whereas the magnetic field variations detected by the VEGA spacecraft were rather smooth (Riedler et al., 1986). In an effort to explain these discrepancies, Galeev et al. (1988) have noted that during the VEGA encounter, the magnetic field was parallel to the relative motion of the two plasma components, giving rise to the firehose instability, which would rapidly decelerate the solar wind plasma.

For the purpose of our global modeling, we consider this transition, referred to as the collisionopause, where enhanced momentum transfer with the outflowing neutrals decelerates the inflowing contaminated solar wind. The cometocentric distance of the collisionopause, along the Sun-comet axis, is determined where the total momentum transfer collision meanfree path between an ion in the inflowing, contaminated solar wind and the outflowing neutral molecules is equal to the radial distance from the nucleus and is given by

$$
\dot{R}_{c}=\frac{\alpha Q_{n}}{4 \pi V_{n}}
$$

where $\alpha$ is the collision cross-section. The outward-streaming cometary neutrals are subject to collisions within this statistically determined scale length, $R_{c}$. Because the solar wind flow is contaminated with multiple ionic species, the momentum transfer cross-sections will vary for the different species, which may lead to some degree of chemical separation. Gringauz et al. (1986a) have also referred to this region as a "chemical boundary" based on the observations at comet Halley of a significant velocity difference between the protons and heavy ions inside the collisionopause. These authors suggest that the velocity difference might be due to the fact that the protons are not coupled to the neutrals as strongly as the heavy ions. In an effort to explain the observed sharpness of the cometopause, Gombosi (1987) has invoked a charge exchange avalanche mechanism that occurs as the solar wind protons are decelerated. 
The Giotto spacecraft identified this transition boundary at a distance of $\approx 1.39 \times 10^{5} \mathrm{~km}$ from comet Halley's nucleus. Assuming the same flare ratio of $\approx 2.15$ as for the bow shock gives the cometocentric collisionopause distance along the Sun-comet axis of $\approx 4.65 \times 10^{4}$ $\mathrm{km}$. If the average ion in the solar wind at this distance is assumed to be $\mathrm{H}_{2} \mathrm{O}^{+}$, then Equation (23) gives $R_{c} \approx 5.5 \times 10^{4} \mathrm{~km}$, if we take $Q_{n} \approx 6.9 \times 10^{29} \mathrm{~mol} / \mathrm{sec}, V_{n} \approx 1 \mathrm{~km} / \mathrm{sec}$, and $\alpha \approx 10^{-14} \mathrm{~cm}^{2}$. The heliocentric variation of $R_{c}$ is shown in Figure 9 using the $Q_{n}(d)$ and $V_{n}(d)$ determined earlier.

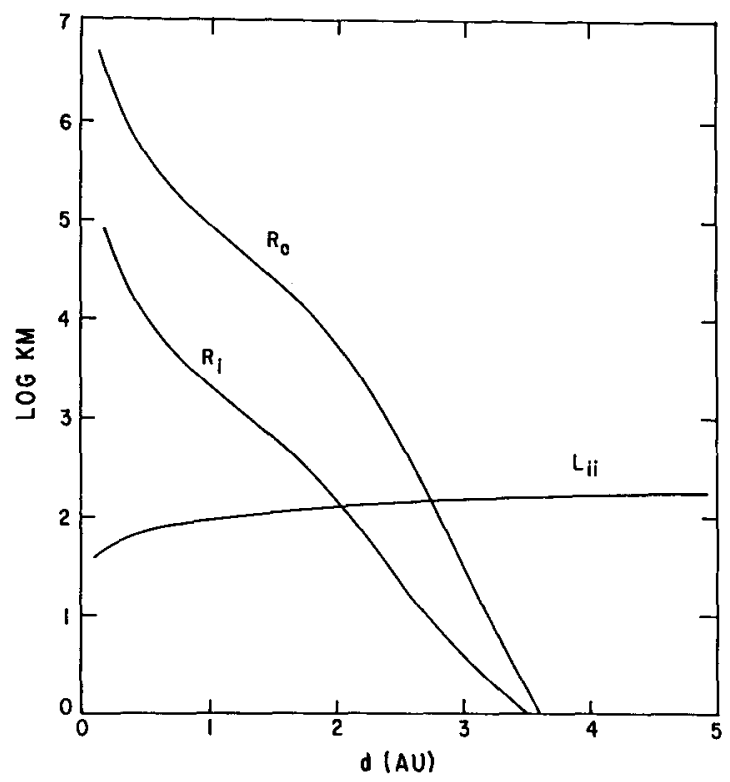

Figure 9. Heliocentric variation of the collisionopause, $R_{c}$, the ionopause, $R_{i}$, and the cometary ion Larmor radius near the ionopause, $L_{i i}$.

Inside the collisionopause, the solar wind decelerates rapidly and also cools due to charge exchange of energetic ions formed upstream with the cool cometary neutrals (Wallis, 1973). This leads to an increase of the magnetic field to form a magnetic barrier region where the solar wind plasma pressure is converted to magnetic pressure.

\subsection{IONOPAUSE}

At the inner edge of the magnetic barrier, a tangential discontinuity interface (hereafter loosely referred to as the cometary ionopause) separates the contaminated solar wind plasma from the purely cometary plasma. The current concept of the cometary ionopause, which has generally been confirmed by the Giotto results at comet Halley, is based on the early model of Ip and Axford (1982). Realizing the strong ion-neutral coupling in the cometary ionosphere, these authors determined that an ionopause would form where the magnetic tension in the curved magnetic field lines at the inner edge of the magnetic barrier is balanced by the drag force exerted by the outflowing cometary neutrals on the stagnant 
ions in this region. It is now generally agreed (Flammer et al., 1987; Mendis et al., 1986; Ip and Axford, 1987; Cravens, 1986) that a balance between the magnetic forces and the ionneutral drag force controls the flow in the vicinity of the tangential discontinuity interface, i.e.,

$$
\frac{1}{4 \pi} B_{i} \frac{d B_{i}}{d r} \hat{r}+\frac{1}{4 \pi} \frac{B_{i}^{2}}{R_{i}} \hat{r}=k_{d} n_{i} n_{n} m_{i}\left(u_{n}-u_{i}\right) \hat{r}
$$

where $B_{i}$ is the magnetic field strength just outside the TD, $R_{i}$ is the radius of curvature, $k_{d}$ is the ion-neutral collision rate coefficient, $n_{n}$ and $n_{i}$ are the neutral and ion number densities, and $u_{n}$ and $u_{i}$ are their flow velocities. Assuming that the dominant term in the $J \times B$ magnetic force is the curvature force and using the 2-D model of Flammer et al. (1987), which allows for plasma loss due to dissociative recombination and to flow parallel to the field lines, to calculate the ion density near the ionopause, we get

$$
R_{i}^{2}=\frac{4 \pi k_{d} m_{i} u_{n} n_{n o} R_{n}^{2}}{B_{i}^{2}} \times\left(-\frac{u_{n o}}{\pi k_{R}}+\left(\frac{u_{\|}^{2}}{\pi^{2} k_{R}^{2}}+\frac{n_{n o} R_{n}^{2}}{k_{R} \tau_{i}}\right)^{1 / 2}\right)
$$

where $R_{n}$ is the nuclear radius, $n_{n o}$ is the neutral density at the surface, $k_{R}$ is the average electron dissociative recombination coefficient, and $u_{\|}$is the plasma flow velocity parallel to the field lines. Assuming that the magnetic field strength just ahead of the ionopause, near the subsolar point, results from the conversion of the solar wind dynamic pressure into magnetic pressure, we obtain the heliocentric variation of the ionopause radius, $R_{i}$ as shown in Figure 9.

Using Equation (25) to predict the ionopause location with the observed values during the Giotto encounter of $Q_{n} \approx 6.9 \times 10^{29} \mathrm{~mol} / \mathrm{sec}$ and $B_{i, \text { (inbound) }} \approx 40 \gamma$ and taking $u_{\|} \approx 1 \mathrm{~km} / \mathrm{sec}, k_{R} \approx 5 \times 10^{-7} \mathrm{~cm}^{3} \mathrm{~s}^{-1}, k_{d} \approx 10^{-9} \mathrm{~cm}^{3} \mathrm{~s}^{-1}, \tau_{i} \approx 2 \times 10^{6} \mathrm{sec}$, we get $R_{i,(\text { inbound })} \approx 4335 \mathrm{~km}$. A somewhat higher magnetic field strength was measured after exiting the magnetic field-free cavity of $B_{i,(\text { outbound })} \approx 50 \gamma$, which, using the same parameters, gives $R_{i \text {,(outbound) }} \approx 3470 \mathrm{~km}$. These results are in reasonable accord with the observed Giotto ionopause crossings of $\approx 4700 \mathrm{~km}$ inbound and $\approx 3900 \mathrm{~km}$ outbound (Neubauer, 1986). Similar model calculations for the ionopause location and magnetic field profile just outside the ionopause have been performed by Ip and Axford (1987) and Cravens (1986) by neglecting the flow along the field lines and assuming photochemical equilibrium to determine the ion density. Cravens retains only the magnetic pressure gradient term in Equation (24), whereas Ip and Axford retain both magnetic terms. Haerendel (1987) and Eviatar and Goldstein (1988) have included the effects of the plasma pressure gradient and mass-loading to calculate the magnetic field morphology. The estimated position of the ionopause in all of these models is comparable and in agreement with the observations, which emphasizes that the dominant process responsible for the formation of the ionopause is the balance between the ion-neutral drag force and the magnetic forces.

The condition for a well-defined ionopause is given by the condition that

$$
R_{i} \geq L_{i i}\left(=\frac{c}{e B_{i}}\left(k T_{2} m_{i}\right)^{1 / 2}\right)
$$

where $L_{i i}$ is the Larmor radius of a cool cometary ion just ahead of the ionopause, and $T_{2}$ is the temperature in that region. The variation of $L_{i i}$ with $\mathrm{d}$ is shown in Figure 9 . It can 
be seen that for a Halley-type comet, the inequality required for a well-defined ionopause given by Equation (26) is satisfied only when $d \leq 2.2 \mathrm{AU}$.

The clearest signature of the ionopause at comet Halley lies in the magnetometer measurements, which showed a well-defined magnetic field free region around the cometary nucleus. The detection of the magnetic field free cavity suggests a stable boundary, impenetrable to the IMF. Ershkovich and Mendis (1986) examined the effects of the outflowing cometary neutrals on the stability of the cometary ionopause. Because the radially flowing neutrals have a finite velocity component normal to the interface, the neutral drag on the stagnant ions gives rise to an interchange instability, referred to as the drag instability. Ershkovich et al. $(1986,1988)$ have treated the cometary ionopause as a tangential discontinuity and shown that it is subject to various magnetohydrodynamic instabilities, namely, the Kelvin-Helmholtz instability, the fluting instability and the "drag" instability. In a more recent analysis, Ershkovich et al. (1989) have considered an overturning instability at the ionopause, similar to that which occurs in unstable atmospheres. For parameters reflecting the conditions surrounding the ionopause at comet Halley, the growth rate of these instabilities is sufficiently low compared with the convection time along the boundary so that significant growth of these waves is not expected.

\subsection{INNER SHOCK}

The existence of an inner shock inside the cometary ionopause has been postulated in order to decelerate the supersonic cometary ions and divert them into the tail (Wallis and Dryer, 1976). While the earlier models suggested a hydrodynamic shock (Houpis and Mendis, 1980; Baranov and Lebedev, 1981), the possibility of an electrostatic shock has now been recognized due to the increased electron pressure in this region (Cravens et al., 1984; Korosmezy et al., 1987; Mendis et al., 1989). A preliminary study of an electrostatic shock of this nature performed by Omidi et al., (1989) using a 1-D hybrid simulation, indicates that the drag force of the outflowing neutrals on the ions can greatly affect the structure of this shock. The measurements at comet Halley showed no direct evidence for the existence of an inner shock of either type; the cometary ions had a radial velocity of $\approx 1 \mathrm{~km} / \mathrm{sec}$ all the way out to the boundary of the magnetic field-free cavity (Schwenn et al., 1987) and a large electron temperature gradient was not observed inside the cavity. However, a population of hot ions moving in the anti-sunward direction was observed inside the ionopause by the Giotto Ion Mass Spectrometer (IMS) (Goldstein et al., 1987), which these authors suggest could be due to a tailward flow of ions that have been deflected and heated near the magnetic cavity boundary. Further analysis of the Giotto IMS data (Goldstein et al., 1989), has shown that a thin, density spike exists at the inner edge of the ionopause. In agreement with these observations, Cravens' (1989) numerical magnetohydrodynamic calculations predict a thin, pile-up of ions just inside the cavity boundary, in a "cavity transition layer", with recombination the primary loss mechanism. Cravens (1989) identifies the inner edge of the cavity transition layer where the ionospheric flow goes subsonic, as an "inner shock", although according to his calculations, the shock thickness is comparable to the cavity transition boundary layer itself and the flow cannot be diverted toward the tail in such a narrow layer. Future analysis is clearly needed in order to understand the unique characteristics of the inner shock, if it exists. Flammer et al. (1989) have recently shown that particle kinetic effects are important in determining the structure of the ionopause 
transition layer. Both the models of Cravens (1989) and Flammer et al. (1989) are onedimensional and clearly an accurate study of the inner shock requires a two-dimensional treatment.

\section{Conclusion}

In this review, simple models have been developed to describe the characteristic plasma boundaries that form in the solar wind interaction with cometary atmospheres. From these models, we can determine the location of these boundary regions relative to the nucleus as a function of the comet's heliocentric distance, and thus make predictions as to where in the comet's orbit they are likely to form. The cometary bow shock, the collisionopause/cometopause, and the ionopause were identified in the plasma and field measurements at Halley's comet, and there is fairly good agreement between the measured and predicted boundary locations. The transition from a shock-free flow to one with a fully developed, collisionless shock is found to take place around $d \approx 2.48$ to $2.6 \mathrm{AU}$. The developing cometary atmosphere becomes sufficiently dense enough to be able to form an ionopause only when $d \leq 2.2 \mathrm{AU}$. These results apply for a comet of similar source strength as comet Halley, so the heliocentric distances at which the transitions take place will be greater/less for a less/more active comet.

\section{References}

Amata, E., Formisano, V., Cerulli-Irelli, R., Torrente, P., Johnstone, A.D., Coates, A., Wilken, B., Jockers, K., Winningham, J.D., Bryant, D., Borg, H., and Thomsen, M. (1986). 'The cometopause region at comet Halley,' ESA SP-250, Vol. 1, 213-218.

Balsiger, H., Altwegg, K., Buhler, F., Geiss, J., Ghielmetti, A.G., Goldstein, B.E., Goldstein, R., Huntress, W.T., Ip, W.-H., Lazarus, A.J., Meier, A., Neugebauer, M., Rettenmund, U., Rosenbauer, H., Schwenn, R., Sharp, R.D., Shelley, E.G., Ungstrup, E., and Young, D.T. (1986). 'Ion composition and dynamics at comet Halley,' Nature, $321,330-335$.

Balsiger, H., Altwegg, K., Buhler, F., Fuselier, S.A., Geiss, J., Goldstein, B.E., Goldstein, R., Huntress, W.T., Ip, W.-H., Lazarus, A.J., Meier, A., Neugebauer, M., Rettenmund, U., Rosenbauer, H., Schwenn, R., Shelley, E.G., Ungstrup, E., and Young, D.T. (1987). 'The composition and dynamics of cometary ions in the outer coma of $\mathrm{P} /$ Halley,' Astron. Astrophys., 187, 163.

Baranov, V.B., Zaitsev, N.A., and Lebedev, M.G. (1986). 'Model of solar wind interaction with atmospheres of comets,' Astron. Zurn., 63, 170.

Biermann, L., Brosowski, B., and Schmidt, H.U. (1967). 'The interaction of the solar wind with a comet,' Solar Phys., 1, 254-283.

Cravens, T.E. (1986). 'Physics of the cometary contact surface,' ESA SP-250, Vol. 1, $241-246$.

Cravens, T.E. (1989). 'A magnetohydrodynamical model of the inner coma of comet Halley,' J. Geophys. Res., 94, 15025. 
Ershkovich, A.I., and Flammer, K.R. (1988). 'Nonlinear stability of the dayside cometary ionopause,' Astrophys. J., 311, 1031.

Ershkovich, A.I., and Mendis, D.A. (1986). 'Effects of the interaction between plasma and neutrals on the stability of the cometary ionopause,' Astrophys. J., 302, 1101.

Ershkovich, A.I., Flammer, K.R., and Mendis, D.A. (1986). 'Stability of the sunlit cometary ionopause,' Astrophys., 87, 1031.

Ershkovich, A.I., McKenzie, J.F., and Axford, W.I. (1989). 'Stability of a cometary ionosphere/ionopause determined by ion-neutral friction,' Astrophys. J., submitted.

Eviatar, A., and Goldstein, B.E. (1988). 'A unidimensional model of the comet ionopause structure,' J. Geophys. Res., 93, 1759.

Flammer, K.R., Mendis, D.A., and Houpis, H.L.F. (1987, in press). 'The variable nature of the solar wind interaction with comet Halley as it approaches the sun,' in Proc. STIP Symposium on Retrospective Analysis and Future Coordinated Intervals, Los Diablerets, Switzerland, D. Reidel Pub. Co.

Flammer, K.R., Omidi, N., and Quest, K.B. (1989). 'The structure of the cometary ionopause,' AGU Fall Meeting, San Francisco, EOS, 70, 1181.

Galeev, A.A. (1986). 'Theory and observations of solar wind/cometary plasma interaction processes,' ESA SP-250, Vol. 1, 3.

Galeev, A.A. (1987). 'Encounters with comets: Discoveries and puzzles in cometary plasma physics,' Astron. Astrophys., 187, 12-20.

Galeev, A.A., and Lipatov, A.S. (1984). 'Plasma processes in cometary atmospheres,' Adv. Space Res., 4, 229-237.

Galeev, A.A., Cravens, T.E., and Gombosi, T.I. (1985). 'Solar wind stagnation near comets,' Astrophys. J., 289, 807-819.

Galeev, A.A., Gringauz, K.I., Klimov, S.I., Remizov, A.P., Sagdeev, R.Z., Savin, S.P., Sokolor, A.Yu., Verigin, M.I., Szego, K., Tatrallyay, M., Grard, R., Yerosenko, Ye.G., Mogilevsky, M., Riedler, W., and Schwingenschuh, K. (1988). 'Physical processes in the vicinity of the cometopause interpreted on the basis of plasma, magnetic field, and plasma wave data measured on board the Vega 2 spacecraft,' J. Geophys. Res., 93, $7527-7531$.

Gazis, P.R., and Lazarus, A.J. (1982). 'Voyager observations of solar wind proton temperature: 1-10 AU,' Geophys. Res. Lett., 9, 431.

Goldstein, B.E., Altwegg, K., Balsiger, H., Fuselier, S.A., Ip, W.-H., Meier, A., Neugebauer, M., Rosenbauer, H., and Schwenn, R. (1989). 'Observations of a shock and a recombination layer at the contact surface of comet Halley,' J. Geophys. Res., $94,17251$.

Goldstein, R., Young, D.T., Balsiger, H., Buehler, F., Goldstein, B.E., Neugebauer, M., Rosenbauer, H., Schwenn, R., and Shelley, G.G. (1987). 'Hot ions observed by the Giotto Ion Mass Spectrometer at the comet P/Halley contact surface,' Astron. Astrophys., 187, 220.

Gombosi, T.I. (1987). 'Charge exchange avalanche at the cometopause,' Geophys. Res. Lett., $14,1174$. 
Gringauz, K.I., Gombosi, T.I., Remizov, A.P., Apathy, I., Szemerey, I., Verigin, M.I., Denchikova, L.I., Dyachkov, A.V., Keppler, E., Klimenko, N., Richter, A.K., Somogyi, A.J., Szego, K., Szendro, S., Tatrallyay, M., Verga, A., and Vladimirova, G.A. (1986a). 'First in situ plasma and neutral gas measurements at comet Halley,' Nature, 321, 282.

Gringauz, K.I., Gombosi, T.I., Tatrallyay, M., Verigin, M.I., Remizov, A.P., Richter, A.K., Apathy, I., Szemerey, I., Dyachkov, A.V., Balakina, O.V., and Nagy, A.F. (1986b). 'Detection of a new chemical boundary at comet Halley,' Geophys. Res. Lett., 13, 613.

Haerendel, G. (1987). 'Plasma transport near the magnetic cavity surrounding comet Halley,' Geophys. Res. Lett., 14, 673.

Houpis, H.L.F., and Mendis, D.A. (1980). 'Physicochemical and dynamical processes in cometary ionospheres - 1. The basic flow profile,' Astrophys. J., 239, 1107-1118.

Ip, W.-H. (1989). 'On charge exchange effect in the vicinity of the cometopause of comet Halley,' Astrophys. J., 343, 946.

Ip, W.-H., and Axford, W.I. (1982). 'Theories of physical processes in cometary comas and ion tails,' in Comets, L.L. Wilkening (ed.), University of Arizona Press, Tucson, Arizona, p. 588 .

Ip, W.-H., and Axford, W.I.'(1987). 'The formation of the magnetic-field-free cavity,' Nature, 325, 418 .

Korosmezey, A., Cravens, T.E., Gombosi, T.I., Nagy, A.F., Mendis, D.A., Szego, K., Gribov, B.E., Sagdeev, R.Z., Shapiro, V.D., and Shevchenko, V.I. (1987). 'A comprehensive model of cometary ionospheres,' J. Geophys. Res., 92, 7331.

Mendis, D.A., and Flammer, K.R. (1984). 'The multiple modes of interaction of the solar wind with a comet as it approaches the sun,' Earth, Moon and Planets, 31, 301.

Mendis, D.A., Houpis, H.L.F., and Marconi, M.L. (1985). 'The physics of comets,' Fundamentals of Cosmic Physics, 10, 1.

Mendis, D.A., Smith, E.J., Tsurutani, B.T., Slavin, J.A., Jones, D.E., and Siscoe, G.L. (1986). 'Comet-solar wind interactions: Dynamical length scales and models,' Geophys. Res. Lett., 13, 239.

Mendis, D.A., Flammer, K.R., Reme, H., Sauvaud, J.A., D'Uston, C., Cotin, F., Cros, A., Anderson, K.A., Carlson, C.W., Curtis, D.W., Larson, D.E., Lin, R.P., Mitchell, D.L., Korth, A., and Richter, A.K. (1989). 'On the global nature of the solar wind interaction with comet Halley,' Annales Geophysicae, 7, 99.

Neubauer, F.M. (1986). 'Giotto magnetic field results on the magnetic field pile-up region and the cavity boundaries,' ESA SP-250, Vol. 1, 35.

Neubauer, F.M. (1987). 'Giotto magnetic field results on the boundaries of the pile-up region and the magnetic cavity,' Astron. Astrophys., 187, 73-79.

Neubauer, F.M. (1988). 'The ionopause transition and boundary layers at comet Halley from Giotto magnetic field observations,' J. Geophys. Res., 93, 7272-7281.

Neugebauer, M., Slavin, J., and Ip, W.-H. (1985). A Plasma Model for Comet Kopff, JPL internal document D-2524, pp. 1-20.

Ogino, T., Walker, R.J., and Ashour-Abdalla, M. (1988). 'A three-dimensional MHD simulation of the interaction of the solar wind with comet Halley,' J. Geophys. Res., 93, 9568-9576. 
Omidi, N., and Winske, D. (1986). 'Simulation of the solar wind interaction with the outer regions of the coma,' Geophys. Res. Lett., 13, 397.

Omidi, N., and Winske, D. (1987). 'A kinetic study of solar wind mass loading and cometary bow shocks,' J. Geophys. Res., 92, 13409-13426.

Omidi, N., Winske, D., and Quest, K.B. (1989). 'The effect of ion/neutral collisions on the structure of electrostatic shocks: Application to cometary inner shocks,' EOS, 70, 384.

Riedler, W., Schwingenschuh, K., Yeroshenko, Ye.G., Styashkin, V.A., and Russell, C.T. (1986). 'Magnetic field observations in comet Halley's coma,' Nature, 321, 288-289.

Reme, H. (1990, in press). 'Regions of interaction between the solar wind plasma and the plasma environment of comets,' in Comet Halley 1986 World-Wide Investigations, Results, and Interpretations, E. Horwood Ltd., Chichester, England.

Sagdeev, R.Z., Shapiro, V.D., Shevchenko, V.I., and Szego, K. (1986). 'MHD turbulence in the comet solar wind interaction region,' Geophys. Res. Lett., 13, 85-88.

Schmidt, H.U., and Wegmann, R. (1976). 'Interaction of interplanetary magnetic fields with cometary plasma,' Max-Planck-Institut für Plasmaphysik, Garching, IPP6-147.

Schmidt, H.U., and Wegmann, R. (1982). 'Plasma flow and magnetic fields in comets,' in Comets, L.L. Wilkening (ed.), University of Arizona Press, Tucson, Arizona, pp. $538-560$.

Schwenn, R., Ip, W.-H., Rosenbauer, H., Balsiger, H., Buhler, F., Goldstein, R., Meier, A., and Shelley, E.G. (1986). 'Ion temperature and flow profiles in comet Halley's close environment,' Astron. Astrophys., 187, 160.

Vaisberg, O.L. (1988). 'Density distribution of heavy ions in the head of Halley's comet,' Cos. Phys., 26, 9.

Wallis, M.K. (1973). 'Weakly shocked flows of the solar wind through atmospheres of comets and planets,' Planet. Space Sci., 21, 1647.

Wallis, M.K., and Dryer, M. (1976). 'Sun and comets as sources in an external flow,' Astrophys. J., 205, 895-899.

Wallis, M.K., and Dryer, M. (1985). 'Decay of the cometary bow shock,' Nature, 318, 646.

Winske, D., Wu, C.S., Li, Y.Y., Mou, Z.Z., and Guo, S.Y. (1985). 'Coupling of newborn ions to the solar wind by electromagnetic instabilities and their interaction with the bow shock,' J. Geophys. Res., 90, 2713.

Wu, C.S., and Davidson, R.C. (1972). 'Electromagnetic instabilities produced by neutral particle ionization in interplanetary space,' J. Geophys. Res., 77, 5399. 